\title{
Ambiente Athena: da concepção à utilização de recursos educacionais digitais granulares
}

\author{
M. Alinne F. de Brito ${ }^{1}$, Rayssa A. Hitzschky¹, Carlos Sergio da S. Marinho', Cintia A. \\ Lima ${ }^{1}$, Fernando A. B. dos Santos Junior ${ }^{1}$, M. Silvania M. X. de Souza ${ }^{1}$, Amanda L. \\ Girão $^{1}$, Carolina Morais R. Silva ${ }^{1}$ 'M. Fátima C. de Souza ${ }^{1}$, Márcia D. Medeiros ${ }^{1}$, J. \\ Aires de Castro Filho ${ }^{1}$ \\ 1 Instituto UFC Virtual - \\ Universidade Federal do Ceará (UFC) Campus do Pici - Bloco 901 - $1^{\circ}$ andar - CEP \\ 60455-760 - Fortaleza - CE \\ \{alinnefbrito; hitzschkyrayssa, sergio.marinho94; \\ arrucin; ofernandosantosjunior; silvaniamarques; amapoet@1; \\ carolina.morais.ufc\} @gmail.com \{fatimasouza; marcia; \\ aires\}@virtual.ufc.br
}

\begin{abstract}
Many are the initiatives undertaken in the search for promoting the use of digital educational resources (DER) in schools. However, teachers face obstacles in integrating the DER due to limited access to the Internet, the size and lack of curriculum guidelines for the use of many resources. The Athena environment was created with the proposal to reduce some of these barriers by providing granular DER in the areas of Portuguese and Mathematics, which were cataloged from the National System Reference Matrix Basic Education Assessment (SAEB) and its descriptors. Moreover, all the resources, and the environment itself, can be saved in auxiliary devices (USB drives up to 4 GB) and used in the classroom.
\end{abstract}

Resumo. Muitas são as iniciativas empreendidas na busca por fomentar o uso dos recursos educacionais digitais (RED) no âmbito escolar. Entretanto, os docentes enfrentam obstáculos na integração dos RED devido ao pouco acesso à Internet, ao tamanho e à falta de diretrizes curriculares para o uso de muitos recursos. $O$ Ambiente Athena surge com a proposta de minimizar alguns desses entraves, através da disponibilização de RED granulares nas áreas de Língua Portuguesa e Matemática e que foram catalogados a partir da matriz de referência do Sistema Nacional de Avaliação da Educação Básica (SAEB) e seus descritores. Ademais, todos os recursos, além do próprio ambiente, podem ser salvos em dispositivos auxiliares (pendrives de até 4Gb) e utilizados localmente em sala de aula.

\section{Athena e seu cenário de uso}

Os recursos educacionais digitais (RED) caracterizam-se como oportunidades ao ensino e à aprendizagem, no que diz respeito às diferentes formas de tratar o conteúdo educacional. No entanto, apesar das potencialidades oferecidas por esses instrumentos, ainda existem inúmeros entraves que inviabilizam a utilização desses recursos por parte do professor. [ROSA, 2013] 
Dentre os obstáculos existentes, é possível destacar a inadequação da infraestrutura referente à conexão de banda larga e a falta de suporte técnico no que concerne ao tamanho dos recursos. Esses obstáculos foram detectados a partir do Projeto Um Computador Por Aluno $^{1}$ (Projeto UCA), fomentado pela Universidade Federal do Ceará (UFC) e implementado em 2007. Essa iniciativa teve como objetivo principal, a inserção de laptops educacionais em escolas da rede pública de ensino.

O Projeto UCA teve como referência o projeto One Laptop Per Child (OLPC), desenvolvido em meados de 2000, por Nicholas Negroponte, pesquisador do Massachussets Institute of Technology (MIT). Este teve como objetivo distribuir um computador portátil de custo reduzido para crianças em idade escolar de países em desenvolvimento a fim de fomentar a inclusão digital. Em contrapartida, o Projeto UCA diferenciou-se da proposta de Negroponte a partir da distribuição de laptops para alunos, usando-os no contexto escolar, em vista de proporcionar experiências pedagógicas inovadoras e possibilidades para a Educação. [CASTRO-FILHO; MAIA; SILVA 2015, p. 11-12]

No intuito de minimizar tais obstáculos, o Ambiente Athena surge com o intuito de fornecer aos professores e à comunidade escolar, recursos de qualidade técnica e pedagógica das áreas de Língua Portuguesa e Matemática, previamente selecionados e catalogados conforme os critérios da matriz de referência do Sistema Nacional de Avaliação da Educação Básica (SAEB) e seus descritores. Além disso, com vista à granularidade (tamanho) dos RED, o Ambiente prioriza materiais que possam ser armazenados em dispositivos auxiliares e utilizados localmente. Os descritores são de grande relevância para o contexto educacional por refletirem as competências e as habilidades esperadas dos educandos ao longo de sua trajetória escolar [BRASIL 2011, p. 18].

Uma das características mais relevantes do Ambiente é a possibilidade de armazenar todo o seu conteúdo em dispositivos de memória auxiliares (usb drives) de até 4GB. Isso minimiza as dificuldades docentes acerca da utilização dos recursos do ambiente sem depender do acesso à Internet. Outro ponto de destaque está relacionado à catalogação dos RED, considerando-se a descrição detalhada dos recursos em seus metadados, incluindo, assim, área de conhecimento, tema do recurso, ano indicado e os descritores associados à matriz de referência citada anteriormente.

Desse modo, o Ambiente Athena apresenta recursos de diferentes tipos e formatos, contendo animações, simulações, softwares, áudios, vídeos e hipertextos. O repositório também disponibiliza documentos nacionais que norteiam a prática docente, assim como recomendações sobre o uso da tecnologia em sala de aula. Os documentos incluem a matriz de referência SAEB, os Parâmetros Curriculares Nacionais (PCN) de Língua Portuguesa e Matemática, as Expectativas de Aprendizagem do estado do Ceará e as Diretrizes Curriculares Nacionais (DCN).

É importante ressaltar que o repositório encontra-se em fase de desenvolvimento e aprimoramento de algumas funções, destacando a inserção de planos de aula associados aos materiais como suporte ao fazer docente. Posteriormente, essa função será implementada, visando proporcionar sugestões de planejamentos pedagógicos que possam ser aplicados na dinâmica de sala de aula.

Com base nisso, o presente documento foi estruturado obedecendo a seguinte ordem de apresentação do Ambiente Athena. Na seção 2, é apresentada a concepção pedagógica do repositório. Na seção 3, o ambiente será apresentado e, por fim, na seção 4, as considerações finais serão feitas.

\footnotetext{
${ }^{1}$ Projeto UCA. <Disponível em: http://www.fnde.gov.br/programas/programa-nacional-de-tecnologiaeducacional-proinfo/proinfo-projeto-um-computador-por-aluno-uca>
} 


\section{Ambiente Athena: da concepção à utilização de recursos granulares}

A concepção e o desenvolvimento do Ambiente Athena perpassaram dois momentos. O primeiro referente à área pedagógica e o segundo referente à área técnica. A concepção desse repositório foi organizada em 6 etapas, das quais as 5 primeiras já foram finalizadas e a sexta etapa ainda encontra-se em processo de construção. As etapas incluíram a busca, seleção, análise, teste e catalogação dos recursos educacionais digitais. Para uma melhor compreensão, descreve-se, na Figura 1, cada uma das etapas desse processo.

\section{0 processo de concepção do Ambiente Athena: \\ Da concepção ao desenvolvimento.}

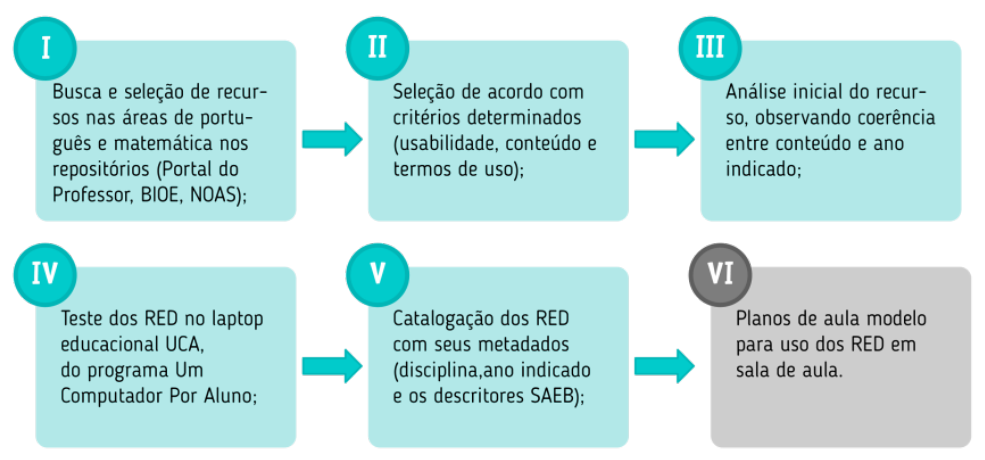

Figura 1. Processo de concepção do Ambiente Athena

Na primeira etapa foram feitas a busca e a seleção de recursos em diferentes repositórios de materiais digitais, das áreas de Língua Portuguesa e Matemática. A busca dos RED foi realizada em repositórios considerados como referência, tais como o Portal do Professor $^{2}$ e o Banco Internacional de Objetos Educacionais (BIOE) ${ }^{3}$. Os critérios para a busca inicial dos RED abrangeram aspectos de uso técnico e pedagógico descritos por Oliveira et al [2001], que vão desde a fundamentação pedagógica, passando pelo conteúdo e finalizando na programação.

Os materiais coletados foram selecionados com base em critérios específicos, conforme a segunda etapa, em termos de usabilidade, conteúdo e licença de uso. Os repositórios citados que serviram como base para a busca dos recursos apresentam licença de uso permitida pelos seus autores, em especial, a licença Creative Commons ${ }^{4}$, possibilitando, assim, o uso dos materiais digitais pelos usuários.

Já na terceira etapa, foi realizada a análise dos recursos, procurando observar a coerência entre o conteúdo e o nível de escolaridade indicado. Na quarta etapa, foram realizados testes de utilização dos RED selecionados no laptop educacional do Projeto UCA. Nessa etapa, os testes foram realizados em diferentes máquinas e sistemas operacionais, dando-se preferência à máquina Classmate $P C$, utilizada pelo seguinte projeto. $\mathrm{O}$ teste dos recursos foi realizado desconectado da rede, observando se existia a possibilidade de utilização dos recursos sem acesso à Internet.

Os materiais catalogados foram selecionados levando-se em consideração a granularidade destes e a sua facilidade de uso local, objetivando suplantar as dificuldades docentes com o uso dos RED em sala de aula. Apesar do conceito de granularidade ser apresentado na literatura como algo que pode ser relacionado tanto ao tamanho quanto a sua

\footnotetext{
${ }^{2}$ Portal do Professor. <Disponível em: http://portaldoprofessor.mec.gov.br/index.html>

3 Banco Internacional de Objetos Educacionais (BIOE). <Disponível em: http://objetoseducacionais2.mec.gov.br/>

${ }^{4}$ Creative Commons. <Disponível em: https://br.creativecommons.org/>
} 
capacidade de reutilização, este trabalho busca relacionar esse conceito com o tamanho dos recursos e, consequentemente, ao esforço de catalogação desses materiais em um repositório aberto [SABBATINI 2012].

Na quinta etapa, definiu-se a forma de catalogação, tomando-se como ponto inicial a adequação dos recursos aos descritores da matriz de referência SAEB e estes deveriam conter em seus metadados, além da disciplina, a origem, o ano e os descritores indicados ao material. É importante salientar que para todos os recursos catalogados, foi levada ainda em consideração a granularidade de cada um desses RED, haja vista que um dos obstáculos em se utilizar tais materiais está relacionado com o tamanho dos recursos e suas possíveis reusabilidades.

Por fim, a sexta e última etapa ainda encontra-se em processo de desenvolvimento, e está relacionada à construção de planejamentos pedagógicos com o uso dos recursos catalogados. Esses planejamentos buscarão tornar o repositório um ambiente de compartilhamento de novos saberes, práticas e metodologias abordadas por professores de diferentes locais em suas aulas.

Com relação ao segundo momento do processo, foram realizadas ações concernentes aos aspectos técnicos, que por sua vez, englobaram atividades de programação e de design, interface do repositório, disposição e visualização dos recursos, arquitetura da informação, bem como as linguagens de programação adotadas.

As ações iniciais foram relacionadas à criação dos elementos que compõem a interface do Ambiente, mais especificamente, à identidade visual do repositório, remetendo aos temas principais que são foco do Ambiente Athena: educação e tecnologia.

O nome Athena foi escolhido por fazer referência à deusa da sabedoria. Assim, o símbolo do Ambiente é representado por uma coruja cibernética, evidenciando a relação entre a questão tecnológica e educacional presente na proposta. Outro ponto de destaque, foi a escolha da família tipográfica. A escolha deu-se pela fonte Federo Regular, por esta transmitir modernidade, com hastes em linha reta e as terminações sem serifa, dando um ar contemporâneo e mais sólido para o logotipo, como apresentado na Figura 2.

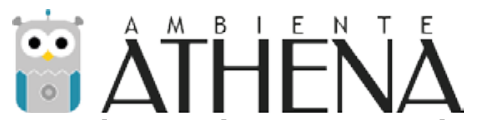

Figura 2. Identidade visual do Ambiente Athena

Além de ser multiplataforma, outro ponto que merece destaque no Ambiente Athena diz respeito à solução adotada para evitar que o uso dos RED fosse dependente da Internet. Para isto, foi feita a escolha pela linguagem de marcação (Extensible Markup Language, doravante XML), utilizadas somente pelo lado do usuário. Dessa forma, a aplicação torna-se portátil, de modo a ser colocada em um pen drive e ser levada a locais mesmo sem acesso à Internet, como em áreas rurais.

A decisão pelo uso da linguagem de marcação $X M L$ para a persistência de dados também foi importante para a independência da aplicação em relação à rede. Os bancos de dados relacionais são comumente utilizados pelo lado do servidor da aplicação ou necessitam de maior trabalho para a sua configuração, quando utilizados do lado do cliente, o que dificultaria a utilização do software de forma off-line.

Além disso, o Ambiente foi concebido para ser multiplataforma, visando o uso dos recursos em diversas plataformas. Desta forma, o repositório foi desenvolvido em linguagens consideradas padrões no que diz respeito ao desenvolvimento para Web: Javascript, Cascading Style Sheets (CSS) versão 3 e Hypertext Markup Language (HTML) versão 5. Esses padrões possibilitam que a página seja acessada em diversos browsers, por exemplo, 
V Congresso Brasileiro de Informática na Educação (CBIE 2016)

Anais dos Workshops do V Congresso Brasileiro de Informática na Educação (CBIE 2016)

Firefox, Chrome, Explorer e Safari, e de vários dispositivos, como desktops, laptops, tablets e smartphones.

\section{Apresentação do Ambiente Athena}

Na página inicial do Athena (Figura 3), encontram-se os ícones de navegação (Início, O Projeto, Recursos, Apoio e Dúvidas), além de opção de download do conteúdo do repositório por completo.

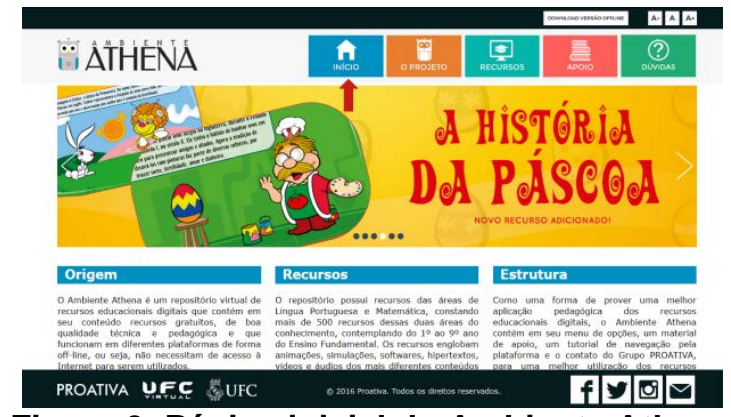

Figura 3. Página inicial do Ambiente Athena

Ainda na página inicial, é possível visualizar os novos recursos que são adicionados ao ambiente, por meio de banners alternados automaticamente ou que podem ser escolhidos através de botões, respectivamente, do lado direito e esquerdo da tela.

Na aba "O Projeto" (Figura 4), podem ser encontradas informações detalhadas acerca da origem do Ambiente Athena, desde a sua concepção.

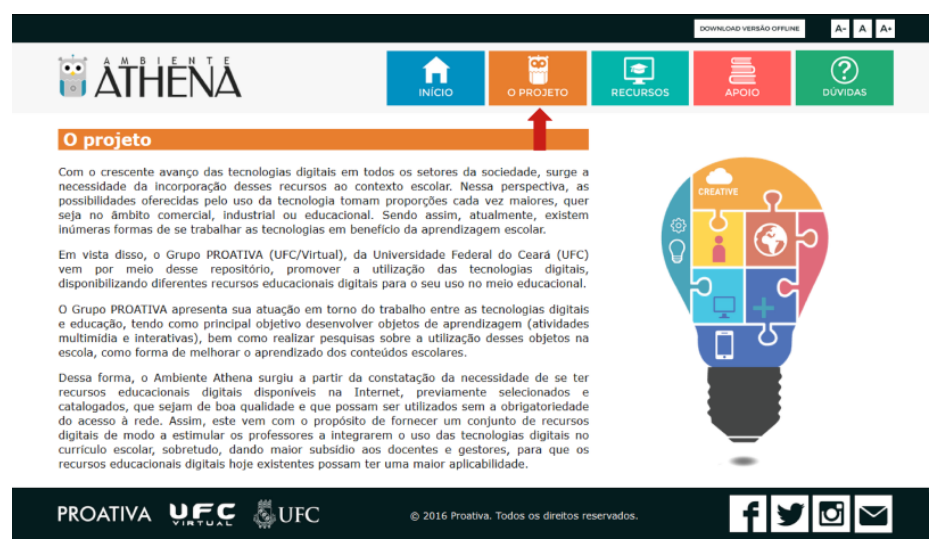

Figura 4. O Projeto

Já na página de "Recursos" (Figura 5), podem ser encontrados os recursos educacionais digitais das áreas de Língua Portuguesa e Matemática, com seu filtro de dados embasado na disciplina, ano e descritores da matriz SAEB, além de informações descritivas sobre cada recurso.

O ícone de descritores, situado abaixo de cada recurso, apresenta os descritores contemplados em cada material, oferecendo, também, o conceito do que é um descritor, visando o entendimento de professores e demais usuários que não possuem familiaridade com esta temática. Os recursos disponibilizados estão em diferentes formatos, como páginas HTML, animações em linguagem Flash, vídeos e áudios. A possibilidade de utilização desses 
V Congresso Brasileiro de Informática na Educação (CBIE 2016)

Anais dos Workshops do V Congresso Brasileiro de Informática na Educação (CBIE 2016)

materiais em diferentes plataformas varia de acordo com a capacidade de cada dispositivo em abri-los. Entretanto, todos eles podem ser utilizados, pelo menos, em desktops e laptops.
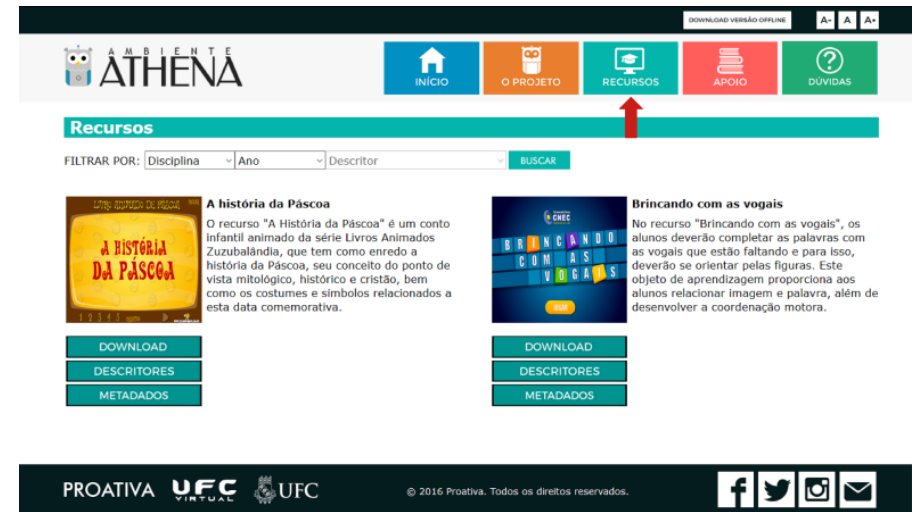

Figura 5. Página referente aos recursos educacionais digitais

Para cada recurso selecionado, têm-se os ícones de download, para possibilitar ao usuário o uso local desses materiais, sem a necessidade de conexão à Internet. Além disso, é possível acessar um link para aprofundar os conhecimentos sobre a matriz de referência SAEB e como se subdividem os descritores da presente matriz (Figura 6).

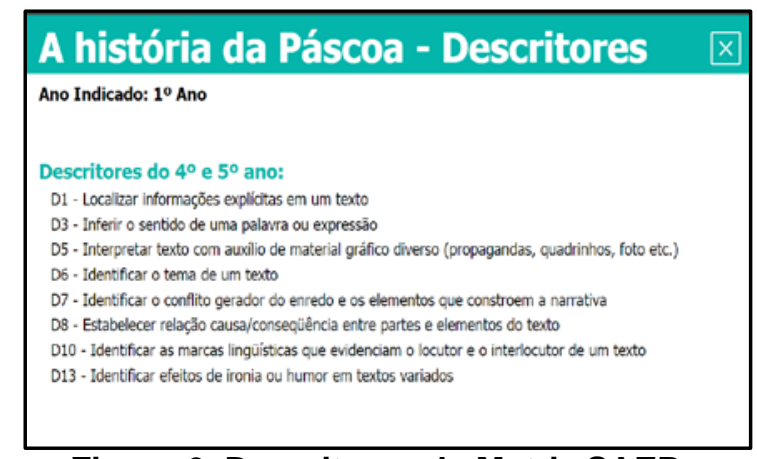

Figura 6. Descritores da Matriz SAEB

Nos metadados de cada RED (Figura 7) são apresentados o tema do recurso escolhido, a sua origem, bem como o idioma, a data de visualização e o ano de criação, procurando, assim, fornecer informações sobre os RED e permitindo, posteriormente, a recuperação destes dados para uma possível reutilização dos recursos educacionais presentes no Ambiente.

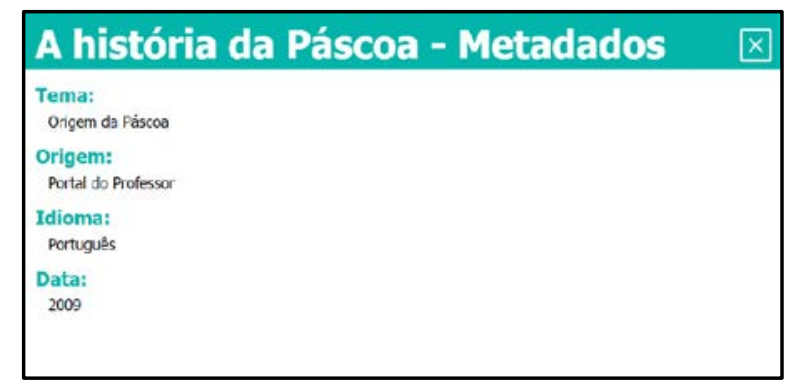

Figura 7. Metadados

$\mathrm{Na}$ “Página de apoio" (Figura 8), são apresentados aos docentes documentos educacionais, incluindo a matriz de referência do Sistema Nacional de Avaliação da 
V Congresso Brasileiro de Informática na Educação (CBIE 2016)

Anais dos Workshops do V Congresso Brasileiro de Informática na Educação (CBIE 2016)

Educação Básica (SAEB), as Diretrizes Curriculares Nacionais da Educação Básica (DCN), os Parâmetros Curriculares Nacionais de Língua Portuguesa e Matemática (PCN) e as Expectativas de Aprendizagem do estado do Ceará, com uma descrição prévia de cada documento e com a opção de baixá-lo no ícone de download. Cabe salientar a importância do fornecimento destes documentos aos professores, visto que estes buscam orientar os docentes no planejamento curricular.

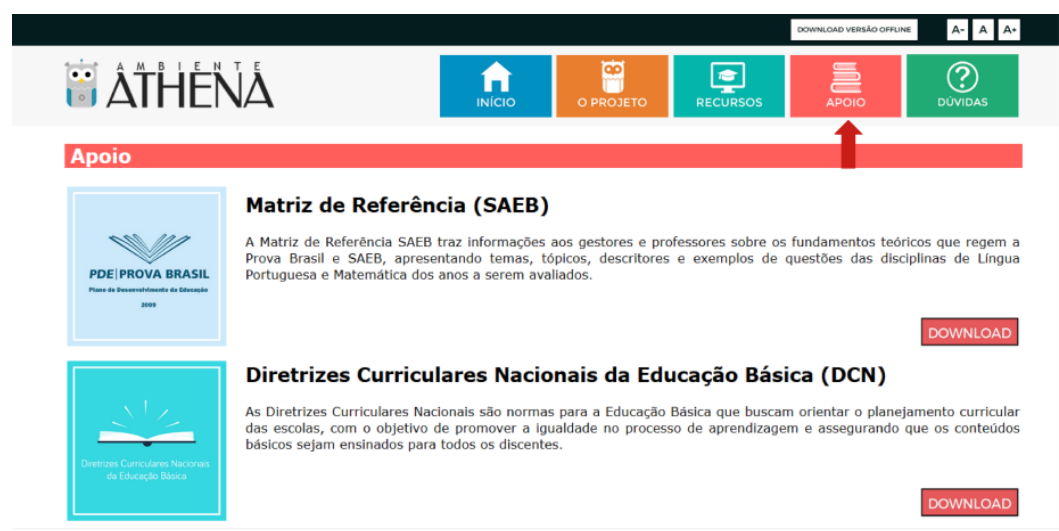

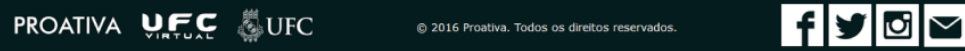

Figura 8. Página de apoio do Ambiente Athena

Visando auxiliar os usuários na navegação do Ambiente, foi criada uma página de “Dúvidas frequentes” (Figura 9), que possui orientações sobre o conteúdo do repositório e um tutorial explicativo de como extrair um arquivo compactado após o download de um recurso.

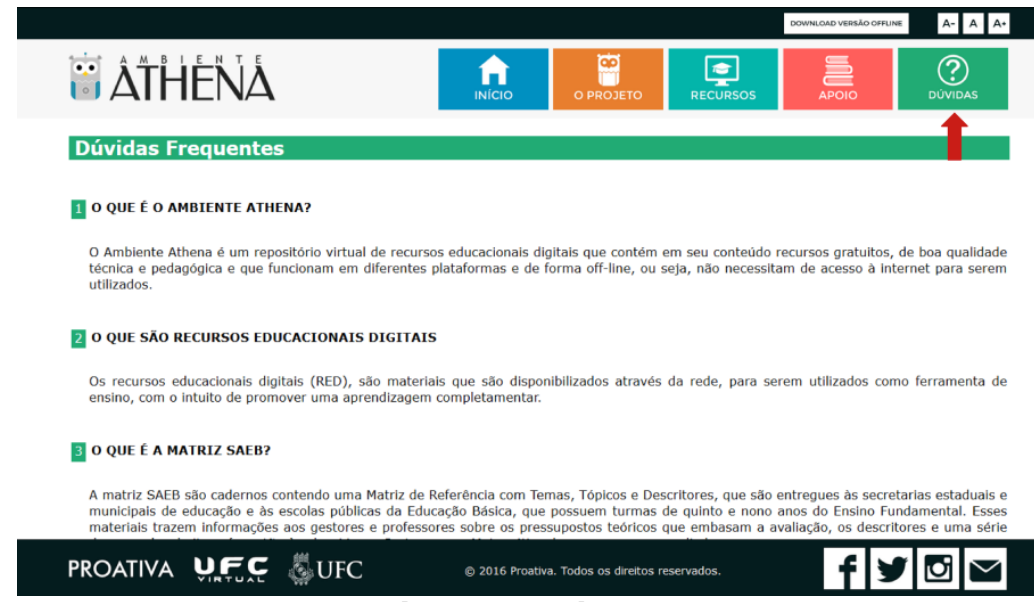

Figura 9. Página de Dúvidas Frequentes

Dessa forma, o Ambiente Athena configura-se como um suporte às práticas pedagógicas desde a sua formulação, a partir das necessidades sentidas por docentes da rede pública de ensino e, sobretudo, a partir da disponibilização de recursos catalogados que abrangem os descritores de uma matriz de referência com abrangência nacional e a possibilidade de armazenamento da totalidade do repositório.

\section{Considerações Finais}

A criação do Ambiente Athena surge como uma iniciativa que busca ampliar a autonomia docente, além de otimizar e potencializar o uso das tecnologias digitais dentro do ambiente 
escolar. Aspectos de diversas naturezas culminaram na concepção do Ambiente em questão, uma delas, em especial, foi a reflexão acerca dos problemas enfrentados por professores do Projeto UCA na utilização dos RED em sua prática docente cotidiana.

Os problemas relatados incidiram em uma expressiva necessidade de construção de um ambiente que apresentasse soluções para o acesso à Internet, obstáculo técnico e estrutural comumente presente nas escolas públicas brasileiras. A partir disso, pensou-se sobre a possibilidade de armazenamento local dos materiais através de dispositivos auxiliares, trazendo, assim, para o cerne da discussão, alternativas para o acesso à rede e suas inúmeras dificuldades.

Alicerçada a essa possibilidade oferecida pelo repositório desenvolvido e como uma forma de enriquecer e aprimorar a ação docente, ponderou-se, paralelamente, sobre a criação de uma catalogação diferenciada a partir de uma matriz de referência que apoiasse o planejamento pedagógico junto ao uso dos recursos.

Buscou-se, ao longo dos momentos de execução, priorizar recursos que se adequassem aos critérios previamente estabelecidos, como forma de equiparar os objetivos determinados às futuras ações realizadas, assim como alinhar possíveis soluções às iniciativas que foram realizadas no âmbito técnico. Todas as ações, apesar de distintas, foram essenciais para a positiva culminância do Ambiente por meio de sua proposta inicial.

É importante destacar que o Ambiente encontra-se em fase de desenvolvimento, com ajustes a serem feitos de âmbito técnico e pedagógico. Dessa forma, como trabalho futuro, pretende-se realizar a inserção de planos de aula, partindo-se do entendimento de que o Ambiente Athena apresenta potencial para se tornar um ambiente de colaboração e formação docente, a partir da criação e disponibilização de planejamentos pedagógicos associados aos atuais recursos presentes ao longo do repositório.

\section{Referências}

Brasil. Ministério da Educação. PDE : Plano de Desenvolvimento da Educação : Prova Brasil: ensino fundamental: matrizes de referência, tópicos e descritores. Brasília : MEC, SEB; Inep, 2008. 200 p. : il.

Castro-Filho, J. A; Maia, D. L. (Org.); Da Silva, Maria Auricélia (Org.). Lições do Projeto Um Computador Por Aluno: estudos e pesquisas no contexto da escola pública. 1 ed. Fortaleza: EdUECE, 2015. v. 1. 331p.

Rosa, Rosemar. VII Encontro de Pesquisa em Educação, II Congresso Internacional, Trabalho docente e Processos Educativos, II Simpósio de Ética em Pesquisa.Trabalho docente: dificuldades apontadas pelos professores no uso das tecnologias. 21 a 25/10/2013.

Sabatini, Marcelo. EM TEIA-Revista de Educação Matemática e Tecnológica Iberoamericana-vol. 3-número 3-2012

Silva, M.;Sala de Aula Interativa a Educação Presencial e à Distância em Sintonia com a Era Digital e com a Cidadania; Evento INTERCOM - Sociedade Brasileira de Estudos Interdisciplinares da Comunicação XXIV Congresso Brasileiro da Comunicação Campo Grande /MS - setembro 2001.

Oliveira, Celina Couto de; COSTA, José Wilson da; MOREIRA, Mercia. Ambientes Informatizados de Aprendizagem - Produção e Avaliação de Software Educativo. Campinas: Papirus, 2001. 